\section{B-cell acute lymphoblastic leukemia as a secondary malignancy following diffuse large B-cell lymphoma}

\author{
Daria Gaut, ${ }^{1}$ Anthony Bejjani, ${ }^{2}$ \\ Joshua Sasine, ${ }^{2}$ Gary Schiller ${ }^{2}$ \\ ${ }^{1}$ Department of Medicine, ${ }^{2}$ Division of \\ Hematology/Oncology, David Geffen \\ School of Medicine, University of \\ California Los Angeles, CA, USA
}

\begin{abstract}
Secondary acute lymphoblastic leukemia (ALL) is a rare disease that has not been well characterized compared with secondary myelodysplastic syndrome or secondary acute myeloid leukemia. We present a report of two patients who developed ALL following complete remission of diffuse large B-cell lymphoma (DLBCL). The first case is more consistent with a therapy-related ALL as a PCR analysis of bone marrow aspirate revealed a distinct clone and the mixed-lineage leukemia gene rearrangement, commonly associated with exposure to topoisomerase II inhibitors. The second case is more consistent with clonal evolution given positive MYC and BCL2 fusion signals in the original diagnosis of DLBCL and the secondary ALL.
\end{abstract}

\section{Introduction}

Secondary malignancies have become an increasing problem for both clinicians and cancer survivors. Secondary acute myeloid leukemia (sAML) evolving from a prior underlying bone marrow disorder or as a product of previous exposure to leukemogenic chemotherapy has been welldescribed. ${ }^{1,2}$ Patients previously treated with alkylating agents typically have a mean latency period of 5-7 years between the primary tumor and development of sAML, that is often associated with complete or partial deletion of chromosome 5 or 7 and/or complex karyotypes. ${ }^{3}$ In contrast, sAML related to prior exposure to topoisomerase II inhibitors is characterized by a shorter latency period (several months to 3 years) and rearrangements at $11 \mathrm{q} 23 .{ }^{3,4}$ In comparison to sAML and secondary myelodysplasia (sMDS), secondary acute lymphoblastic leukemia (sALL) is rare, and has not been well characterized. We present a report of two patients who developed acute lymphoblastic leukemia (ALL) fol- lowing complete remission of diffuse large B-cell lymphoma (DLBCL).

\section{Case Report \#1}

The first patient was a 60 -year-old male with past medical history of psoriasis controlled with cutaneous therapy who originally presented with a new maculopapular rash in August of 2015. A punch biopsy revealed cutaneous lymphoma with a mixture of $\mathrm{CD} 3+$ reactive T-cells and a predominance of $\mathrm{CD} 20+\mathrm{B}$ cells. BCL1 staining was negative, BCL2, BCL6, CD10, and CD21 staining was positive, and $\mathrm{Ki}-67$ revealed an intermediate nuclear proliferation rate compatible with a low-grade lymphoma. On Positron Emission Tomography/ Computed Tomography (PET/CT) scan, he was found to have extensive lymph node and possible bone marrow involvement. Parotid gland dissection and biopsy revealed DLBCL (40-50\%) and follicular lymphoma, World Health Organization (WHO) grade 1-2 (50-60\%) with immunohistochemical (IHC) staining positive for CD20, PAX5, CD10, MYC, BCL2, and BCL6. Bone marrow aspiration and biopsy demonstrated follicular lymphoma in approximately $5 \%$ of marrow elements. The patient was treated with rituximab, cyclophosphamide, doxorubicin, vincristine, and prednisone (R-CHOP) for 6 cycles and achieved a complete remission followed by maintenance rituximab every 3 months. After 8 cycles of rituximab, 2 years after completion of initial therapy, repeat PET/CT scan revealed interval development of several foci of intense fluorodeoxyglucose (FDG) uptake in the bones, suspicious for new osseous lymphoproliferative disease. Bone marrow biopsy revealed B-lymphoblastic leukemia, involving about $90 \%$ of the marrow. Flow cytometric studies showed aberrant expression of partial $\operatorname{dim}$ CD15. Chromosome analysis revealed an abnormal male karyotype $46, \mathrm{XY}, \mathrm{t}(4 ; 11)$ (q21;q23)[10]/46,XY[10] and fluorescence in situ hybridization (FISH) testing confirmed the mixed-lineage leukemia (MLL) rearrangement. A B-cell gene rearrangement/clonality analysis was performed by polymerase chain reaction (PCR) on the bone marrow aspirate and revealed a different clone from that seen in the parotid gland biopsy done at original DLBCL diagnosis. At the time of ALL diagnosis, the patient was asymptomatic with normal complete blood count and negative cerebrospinal fluid (CSF) cytology. He underwent induction with augmented Berlin-FrankfurtMunster (BFM) protocol withholding
Correspondence: Gary Schiller, Hematological Malignancies/Stem Cell Transplantation Unit, David Geffen School of Medicine at UCLA, 10833 Le Conte Ave, Room 42-121 CHS, Los Angeles, CA 90095, USA.

Tel: +1.310.206.5756 - Fax: +1.310.206.5511 E-mail: gschiller@mednet.ucla.edu

Key words: acute lymphoblastic leukemia, diffuse large B-cell lymphoma, secondary malignancy, secondary acute lymphoblastic leukemia, therapy-related acute lymphoblastic leukemia.

Contributions: the authors contributed equally.

Conflict of interest: the authors declare no potential conflict of interest.

Funding: none.

Received for publication: 2 March 2019. Accepted for publication: 13 May 2019.

This work is licensed under a Creative Commons Attribution-NonCommercial 4.0 International License (CC BY-NC 4.0).

(C) Copyright D. Gaut et al., 2019

Licensee PAGEPress, Italy

Hematology Reports 2019; 11:8100

doi:10.4081/hr.2019.8100

asparaginase due to his age, and achieved a complete response. Following consolidation, he underwent allogeneic stem cell transplant with a matched related donor and reduced intensity conditioning of busulfan $0.8 \mathrm{mg} / \mathrm{kg} \mathrm{q} 6 \mathrm{~h} \times 12$ doses (day -10 through -7) and fludarabine $30 \mathrm{mg} / \mathrm{m}^{2} \mathrm{q} 24 \mathrm{~h} \times 5$ doses (day -6 through -2) in addition to prophylactic cranial radiation. Post-bone marrow transplant bone marrow biopsy showed no evidence of disease and chimerism with $>97 \%$ of donor DNA. Currently, the patient is doing well five months post-transplant (2.5 years from original diagnosis of lymphoma).

\section{Case Report \#2}

The second patient was a 58-year-old female with past medical history of basal cell carcinoma who originally presented with multiple palpable masses of the left neck in September of 2015. Lymph node biopsy revealed grade 2 follicular lymphoma with predominately $(60-70 \%)$ follicular architecture and zones of intrafollicular infiltration (CD10, CD20, PAX5, and BCL2 positive). PET/CT revealed extensive disease with FDG uptake in an enlarged 
spleen, multiple enlarged lymph nodes bilaterally, a soft-tissue mass in the region of the porta hepatis/pancreas, and throughout various osseous structures. Bone marrow biopsy showed involvement by follicular lymphoma in $35-40 \%$ of the marrow. The patient was enrolled in the IPI-145 clinical trial, receiving obinutuzumab (antiCD20 monoclonal antibody) in combination with duvelisib (phosphoinositide 3kinase (PI3K) inhibitor). She completed 1 year of therapy with near complete response. Ten months after therapy completion, she developed generalized muscle pain and enlarging left inguinal lymphadenopathy. PET/CT scan showed diffuse disease, including pelvic lymphadenopathy, osseous lesions, and intra and extrathoracic lymphadenopathy. Pelvic mass biopsy revealed follicular lymphoma grade 3B/double-hit DLBCL with FISH analysis positive for MYC and B-cell lymphoma 2 (BCL2) gene rearrangements. She received dose-adjusted etoposide, doxorubicin, vincristine, cyclophosphamide, and prednisone (EPOCH) for six cycles with intrathecal methotrexate (IT-MTX) for CNS prophylaxis and achieved a partial response. She then received two cycles of dexamethasone, high-dose cytarabine, and cisplatin (DHAP) with complete response followed by consolidation with an allogeneic stem cell transplant (matched related donor, reduced intensity conditioning of busulfan 0.8 $\mathrm{mg} / \mathrm{kg} \mathrm{q} 6 \mathrm{~h} \times 12$ doses (days -10 though -7) and fludarabine $30 \mathrm{mg} / \mathrm{m}^{2} \mathrm{q} 24 \mathrm{~h} \times 5$ doses (days -6 through -2)). Two months posttransplant, she developed bony pains. Posttransplant PET/CT revealed intensely FDG avid mild splenomegaly and diffuse, heterogeneous bone marrow uptake with $23 \%$ abnormal circulating mononuclear cells on peripheral smear. Bone marrow biopsy revealed acute B-lymphoblastic leukemia involving $85 \%$ of marrow cellularity, and flow cytometry showed B-lymphoblastic leukemia ( $80 \%$ of total cells) expressing CD19, CD10, and terminal deoxynucleotidyl transferase (TdT). Chromosome analysis revealed an abnormal female complex karyotype with multiple recurrent abnormalities in 19 of 20 cells analyzed: 51, $\mathrm{XX}, \operatorname{der}(5),+\mathrm{i}(6)$, translocations involving chromosomes $(1 ; 5),(8 ; 14)$, and $(14 ; 18)$, addition of chromosome $1,7,11,16$, and 17 , and deletion of chromosome 13. FISH revealed $\mathrm{t}(8 ; 14), \quad+8 \mathrm{q}, \quad \mathrm{t}(14 ; 18)$, and $+14 \mathrm{q}$. Hematologic malignancy sequencing panel was positive for mutations in Cyclin Dependent Kinase Inhibitor 2A (CDKN2A, c.522-1C $>$ A(p.V174_splice) with mutant allele fraction (MAF) $9 \%$ ) and ETS Variant 6 (ETV6, c.218C $>$ T(p.A73V) with MAF $6 \%$ ). She was started on augmented BFM induction without asparaginase but continued to have an increasing blast count and so was transitioned to blinatumumab. Bone marrow biopsy after cycle 1 revealed no leukemia involvement and a new mutation in DNA (cytosine-5)-methyltransferase 3 alpha (DNMT3A, c.928A > T(p. I310F) with MAF 3\%). She completed 2 cycles and received infusion of chimeric antigen receptor T cells (CAR-T cells) (tisagenlecleucel) following conditioning of fludarabine 25 $\mathrm{mg} / \mathrm{m}^{2} /$ day on days $-4,-3,-2$ and cyclophosphamide $250 \mathrm{mg} / \mathrm{m}^{2} /$ day on days $-4,-3,-2$. Three months after CAR T-cellinfusion, PET/CT scan revealed a complete remission and bone marrow biopsy was negative for disease, including detection of minimal residual disease (MRD) by flow cytometry.

\section{Discussion}

sALL is a uncommon disease that has been reported to account for $2-10 \%$ of all cases of ALL. ${ }^{5-9}$ Due its rarity and often underrecognition, the cytogenetic, molecular, and disease characteristics have not been as well-characterized as SMDS and sAML. However, some patterns have been observed. Rearrangements of the MLL gene on chromosome 11 appear to be more common among sALL patients compared to $d e$ novo ALL, ${ }^{7}$ and sALL is often associated with $t(4 ; 11),{ }^{7,10}$ There is also a higher incidence of the pro-B (CD10-negative) immunophenotype with significantly higher expression of CD15 and CD65, compared to de novo CD10-positive ALL. 6,7 Finally, sALL patients are often older at diagnosis and have a lower overall survival than $d e$ novo ALL. 5,11

The pathogenesis of sALL is not fully clear and may be multifactorial. Given the high frequency of the MLL gene rearrangement, also associated with SAML and prior exposure to topoisomerase II inhibitors, many have assumed sALL is therapy-related. 8,9 Other studies have suggested that, given the high incidence of familial malignancy in those patients with SALL, this disease process may be a result of an inherent predisposition to malignancies, as opposed to a result of prior therapy. ${ }^{6,12}$ Lastly, this disease state could be a representation of clonal evolution from a prior malignancy. It is well known that patients with an original presentation of DLBCL or ALL often relapse with the same type of malignancy and such a secondary cancer is clonally related to the original diagnosis. ${ }^{13-15}$ The transformation from a lymphoma to a leukemia would require a clonal relation- ship of mature B cells and immature lymphoblasts through acquisition or silencing of genes in order to produce the immature phenotype. This is less likely, but there is the possibility of leukemia evolution from a common B-cell precursor. The fact that sALL has been documented to occur in the absence of prior chemotherapy lends support for this possibility. ${ }^{12}$

We present a unique report of two patients who developed sALL after therapy and remission of DLBCL. The first case presented is more consistent with a therapyrelated ALL (t-ALL) given the PCR analysis of bone marrow aspirate revealing a distinct clone and the presence of the MLL gene rearrangement. The second case almost certainly represents a clonal evolution given the positive MYC and BCL2 fusion signals in the original diagnosis of DLBCL and the SALL.

There are two other reports in the literature of patients with DLBCL, and even multiple myeloma, that relapsed as ALL. ${ }^{16-}$ ${ }^{18}$ In one study, molecular analysis of the immunoglobulin heavy-chain gene showed that the initial lymphoma and its relapse were clonally related. ${ }^{16}$ Although more common in indolent lymphomas, DLBCL has also been rarely shown to present or progress to a leukemic phase with mature B cells in the peripheral blood, but this is distinct from our presentation of patients with a lymphoblastic phenotype. ${ }^{19,20}$

Our study is also unique in that we present a patient with an ALL characterized by the BCL2/MYC rearrangement, which is rare but has been described previously. ${ }^{21}$ This phenotype has been associated with early treatment failure, ${ }^{21}$ akin to our patient's rising blast count during induction therapy.

\section{Conclusions}

SALL is a distinct entity from de novo ALL that is increasingly being reported. We present an additional two patients with such a phenomenon who relapsed with SALL after treatment and complete remission of DLBCL. Further research is needed to determine the causality of such SALL clones and to assess their clinical implications.

\section{References}

1. Larson RA. Is secondary leukemia an independent poor prognostic factor in acute myeloid leukemia? Best Pract Res Clin Haematol 2007;20:29-37.

2. Bennett JM. Secondary acute myeloid 
leukemia. Leuk Res 1995;19:231-2.

3. Andersen MK, Johansson B, Larsen SO, Pedersen-Bjergaard J. Chromosomal abnormalities in secondary MDS and AML. Relationship to drugs and radiation with specific emphasis on the balanced rearrangements. Haematologica 1998;83:483-8.

4. Leone G, Voso MT, Sica S, et al. Therapy Related Leukemias: Susceptibility, Prevention and Treatment. Leuk Lymphoma 2001;41: 255-76.

5. Giri S, Chi M, Johnson B, et al. Secondary acute lymphoblastic leukemia is an independent predictor of poor prognosis. Leuk Res 2015;39: 1342-6.

6. Pagano L, Pulsoni A, Tosti ME, et al. Acute lymphoblastic leukaemia occurring as second malignancy: report of the GIMEMA archive of adult acute leukaemia. Gruppo Italiano Malattie Ematologiche Maligne dell'Adulto. $\mathrm{Br}$ J Haematol 1999;106:1037-40.

7. Ishizawa S, Slovak ML, Popplewell L, et al. High frequency of pro-B acute lymphoblastic leukemia in adults with secondary leukemia with 11q23 abnormalities. Leukemia 2003;17:1091-5.

8. Tang G, Zuo Z, Thomas DA, et al. Precursor B-acute lymphoblastic leukemia occurring in patients with a history of prior malignancies: is it therapy-related? Haematologica 2012;97:
919-25.

9. Abdulwahab A, Sykes J, Kamel-Reid S, et al. Therapy-related acute lymphoblastic leukemia is more frequent than previously recognized and has a poor prognosis. Cancer 2012;118:39627.

10. Andersen MK, Christiansen DH, Jensen BA, et al. Therapy-related acute lymphoblastic leukaemia with MLL rearrangements following DNA topoisomerase II inhibitors, an increasing problem: report on two new cases and review of the literature since 1992. Br J Haematol 2001;114:539-53.

11. Tang G, Zuo Z, Thomas DA, et al. Precursor B-acute lymphoblastic leukemia occurring in patients with a history of prior malignancies: is it therapy-related? 2012;97:919-25.

12. Ganzel C, Devlin S, Douer D, et al. Secondary acute lymphoblastic leukaemia is constitutional and probably not related to prior therapy. $\mathrm{Br} \mathrm{J}$ Haematol 2015;170:50-5.

13. Melchardt T, Hufnagl C, Weinstock $\mathrm{DM}$, et al. Clonal evolution in relapsed and refractory diffuse large B-cell lymphoma is characterized by high dynamics of subclones. Oncotarget 2016;7:51494-502.

14. Lang F, Wojcik B, Rieger MA. Stem Cell Hierarchy and Clonal Evolution in Acute Lymphoblastic Leukemia. Stem
Cells Int 2015;2015:137164.

15. Jan M, Majeti R. Clonal evolution of acute leukemia genomes. Oncogene 2013;32:135-40.

16. Au WY, Srivastava G, Wong KY, et al. Transformation of diffuse large B-cell lymphoma into pre-B acute lymphoblastic leukemia: clinicopathologic features and clonal relationship. Hum Pathol 2004;35:900-3.

17. Benedek I, Lázár E, Pakucs A, et al. Transformation of Aggressive NonHodgkin Lymphoma in Acute Lymphoblastic Leukemia. J Interdiscip Med 2017;2:72-6.

18. Piszcz J, Bolkun L, Cichocka E, Kloczko J. Secondary acute lymphoblastic leukaemia in a multiple myeloma patient. Contemp Oncol (Poznan, Poland) 2012;16:593-5.

19. Pires PP, Kanegae MY, Rays J, et al. Diffuse large B-cell lymphoma presenting in the leukemic phase. Autops Case Rep 2016;6:41-5.

20. Shuhua Y, Zhong S, Zou D, et al. BCL2 and c-MYC Rearrangements in Leukemic Phase of Diffuse Large B Cell Lymphoma Predicts Central Nervous System Involvement. Blood 2014; 124 .

21. Gu Z, Churchman ML, Roberts KG, et al. PAX5-driven subtypes of B-progenitor acute lymphoblastic leukemia. Nat Genet 2019:1. 